\title{
Decreased NKG2D expression on NK cells correlates with impaired NK cell function in patients with gastric cancer
}

\author{
Hiroaki Saito • Tomohiro Osaki • Masahide Ikeguchi
}

Received: 30 October 2010/Accepted: 18 April 2011/Published online: 31 May 2011

(c) The International Gastric Cancer Association and The Japanese Gastric Cancer Association 2011

\begin{abstract}
Background Although malignant diseases are known to be associated with immune suppression, the detailed mechanisms involved are still unknown. NKG2D is an activating cell surface receptor expressed by natural killer (NK) cells and $\mathrm{CD} 8+\mathrm{T}$ cells, and the engagement of NKG2D is extremely important for NK cell activation. Although decreased NKG2D expression on NK cells is closely related to immune evasion by some cancers, the immunopathological importance of this phenomenon in gastric cancer patients remains unclear.

Methods NKG2D expression on NK cells was determined, using multicolor flow cytometry, to investigate the mechanisms responsible for immune evasion in gastric cancer patients.

Results NKG2D expression on NK cells from gastric cancer patients was significantly lower than that in healthy controls. Also, NKG2D expression in advanced gastric cancer was significantly lower than that in early gastric cancer. NK cells from patients with lymph node metastasis expressed significantly lower levels of NKG2D than the NK cells from those without lymph node metastasis, and NKG2D expression on NK cells in gastric cancer tissue was significantly lower than that of circulating NK cells. NKG2D expression on NK cells obtained from cancer patients was restored after $48 \mathrm{~h}$ in culture with RPMI containing $10 \% \mathrm{AB}$ serum. Furthermore, NKG2D expression on NK cells obtained after surgery was significantly higher than that before surgery.
\end{abstract}

H. Saito $(\bowtie) \cdot$ T. Osaki $\cdot$ M. Ikeguchi

Division of Surgical Oncology, Department of Surgery,

Tottori University School of Medicine, 36-1 Nishi-cho,

Yonago 683-8504, Japan

e-mail: sai10@med.tottori-u.ac.jp
Conclusions Decreased NKG2D expression on NK cells may be one of the key mechanisms responsible for NK cell dysfunction in gastric cancer.

Keywords Gastric cancer - NKG2D - Natural killer cell

\section{Introduction}

Natural killer (NK) cells are effector lymphocytes of the innate immune system that control several types of tumors and microbial infections by limiting their spread and subsequent tissue damage [1]. Since NK cells exhibit natural cytotoxicity against a broad range of human solid tumors without the need for major histocompatibility complex molecule expression by the target cells [2-5], they play an important role in host anticancer defense mechanisms both in vitro [6] and in vivo [7, 8]. Animal studies show that depressed NK cell activity leads to a high incidence of tumor occurrence $[9,10]$. NK cells also play an important role in inhibiting experimental tumor metastasis [11-13]. In humans, an 11-year follow-up epidemiologic survey showed that the level of NK cell activity in peripheral blood was associated with cancer risk in adults; low NK cell activity was associated with increased cancer risk [14].

Gastric cancer is one of the most common malignancies worldwide. Although the prognosis of patients with gastric carcinoma has improved due to the availability of better diagnostic techniques and intra-operative and post-operative care, it still ranks second among all cancer deaths worldwide [15]. Both systemic and local immune defects have been identified in gastric cancer patients [16, 17], and these defects correlate with disease progression and prognosis [18]. Defects in NK cell activity have also been found in patients with gastric cancer [19]. Takeuchi et al. 
demonstrated that low NK cell activity was an independent prognostic indicator in gastric cancer patients [20]. However, the mechanisms responsible for these defects in NK cell function have yet to be defined.

NKG2D is a type II C-lectin-like protein encoded by a gene located next to the NKG2A, NKG2C, and NKG2E genes within the natural killer (NK) gene complex on human chromosome 12p12-p13 and mouse chromosome 6 [21]. NKG2D is an activating cell surface receptor expressed by NK cells, gamma-delta T cells, some cytolytic CD8+ alphabeta $\mathrm{T}$ cells, and NKT cells [22-25]. A recent study demonstrated that tumor-infiltrating and systemic NK cells and CD8 $+\mathrm{T}$ cells often express low levels of NKG2D and are functionally compromised in some cancer patients, indicating that decreased NKG2D expression correlates with a defect in NK cell function [26]. However, the levels of NKG2D expression on NK cells have yet to be determined in gastric cancer patients. Therefore, in this study, we determined the level of NKG2D expression on NK cells from gastric cancer patients to identify the mechanisms responsible for immune evasion in gastric cancer.

\section{Patients and methods}

Patients and healthy controls

Fifty patients treated at Tottori University Hospital and pathologically diagnosed with gastric cancer were enrolled in this study. None of the patients received radiotherapy, chemotherapy, or other medical interventions before surgery. Informed consent for blood donations was obtained for all individuals. Patient characteristics are shown in Table 1. The study included 25 healthy age-matched controls $(62.2 \pm 14.0$ years for the controls vs. $63.3 \pm 12.2$ years for the patients) and each experiment was performed in parallel for the patients and the healthy controls. The clinicopathological findings were determined according to the Japanese classification of gastric carcinoma [27].

Preparation of peripheral blood mononuclear cells

Peripheral blood $(30 \mathrm{ml})$ was drawn from the controls and patients before surgery or chemotherapy and centrifuged on a Ficoll-Paque gradient (Pharmacia, Uppsala, Sweden). Furthermore, peripheral blood was drawn from 10 patients 1 month after surgery to compare NKG2D expression on NK cells between before and after surgery.

Isolation of tumor-infiltrating lymphocytes

Freshly excised tumor tissues were minced, and incubated in collagenase D $(1.5 \mathrm{mg} / \mathrm{ml}$; Wako Pure Chemical
Table 1 NKG2D expression on natural killer (NK) cells and clinicopathologic factors in gastric cancer patients

\begin{tabular}{lclc}
\hline & $\begin{array}{l}\text { No. of } \\
\text { patients }\end{array}$ & $\begin{array}{l}\text { NKG2D } \\
\text { expression (\%) }\end{array}$ & $P$ value \\
\hline Histology & & & \\
$\quad$ Differentiated & 23 & $44.0 \pm 15.6$ & 0.50 \\
$\quad$ Undifferentiated & 27 & $42.7 \pm 12.1$ & \\
Depth of invasion & & & \\
T1 & 32 & $48.4 \pm 10.7$ & 0.0007 \\
T2/T3/T4 & 18 & $34.3 \pm 14.0$ & \\
Lymph node metastasis & & & \\
Absent & 36 & $47.3 \pm 9.8$ & 0.0082 \\
Present & 14 & $33.1 \pm 17.1$ & \\
Lymphatic vessel invasion & & 0.074 \\
Absent & 28 & $46.9 \pm 10.5$ & \\
Present & 22 & $38.7 \pm 16.0$ & \\
Blood vessel invasion & & & \\
Absent & 34 & $44.7 \pm 12.3$ & 0.33 \\
Present & 16 & $40.3 \pm 16.2$ &
\end{tabular}

T1 tumor invasion of the epithelium or lamina propria mucosae or muscularis mucosae or submucosa, $T 2$ tumor invasion of the muscularis propria or the subserosa, T3 penetration of the serosa, T4 invasion of adjacent organs

Industries, Osaka, Japan). Cell suspensions were then filtered through a mesh filter (BD Falcon, Franklin Lakes, NJ, USA).

Flow cytometry analysis

Fluorescent activated cell sorting (FACS) ${ }^{\circledR}$ analysis was performed using a FACSCalibur ${ }^{\mathrm{TM}}$ (Becton-Dickinson, Franklin Lakes, NJ, USA). The following antibodies were used to classify the cells: anti-CD3-PE-Cy5, anti-CD56PE, purified anti-human NKG2D monoclonal antibody $(\mathrm{mAb})$, and fluorescein isothiocyanate (FITC)-labeled goat anti-mouse Ig (BD Pharmingen, Franklin Lakes, NJ, USA). In brief, peripheral blood mononuclear cells $\left(1 \times 10^{5}\right)$ were stained with the titrated antibodies (anti-CD3-PECy5, anti-CD56-PE, purified anti-human NKG2D mAb) in the dark at $4^{\circ} \mathrm{C}$ for $30 \mathrm{~min}$ and washed once. Cells were incubated with the appropriate concentrations of FITClabeled goat anti-mouse Ig in the dark at $4^{\circ} \mathrm{C}$ for $30 \mathrm{~min}$, washed once, and analyzed.

Measurement of soluble MHC class I-related chain gene A (MICA) in sera

The levels of soluble MICA (sMICA) in human sera were measured by enzyme-linked immunosorbent assay (ELISA) using a human MICA ELISA Kit (IMMATICS Biotechnologies, Tubigen, Germany). 
Statistical analysis

Paired $t$-tests or Mann-Whitney $U$-tests were used to determine statistically significant differences between two groups. Correlations were analyzed using the Spearman rank correlation coefficient. The accepted level of significance was $P<0.05$. GraphPad PRISM software (Graphpad Software, San Diego, CA, USA) was used for all statistical analyses.

\section{Results}

NKG2D expression on circulating NK cells in patients with gastric cancer

First, we determined NKG2D expression on circulating NK cells in both healthy controls and gastric cancer patients. NKG2D expression on NK cells from gastric cancer patients (43.3 $\pm 13.7 \% ; n=50)$ was significantly lower than that in the healthy controls $(52.5 \pm 16.9 \% ; n=25$; $P=0.019$; Fig. 1).

Table 1 shows the correlation between NKG2D expression on NK cells and various clinicopathologic factors. NKG2D expression in advanced gastric cancer (T2/ T3/T4 $34.3 \pm 14.0 \%$ ) was significantly lower than that in early gastric cancer (T1 $48.4 \pm 10.7 \% ; P=0.0007)$. NK cells from patients with lymph node metastasis expressed significantly lower levels of NKG2D than the NK cells from those without lymph node metastasis $(33.1 \pm 17.1 \mathrm{vs}$. $47.3 \pm 9.8 ; \quad P=0.0082)$. These results indicate that reduced NKG2D expression on NK cells is closely correlated with disease severity.

We previously demonstrated that NKG2D expression on $\mathrm{CD} 8+\mathrm{T}$ cells was also downregulated in gastric cancer patients [17]. Therefore, we examined the correlation between NKG2D expression on CD8 + T cells and NK cells and found a significant correlation ( $r=0.36, P=0.01$; Fig. 2 ).

NKG2D expression on NK cells in gastric cancer tissues

We then determined the level of NKG2D expression on $\mathrm{NK}$ cells obtained from gastric cancer tissues. NKG2D expression on NK cells from gastric cancer tissues $(38.8 \pm 12.1 \%)$ was significantly lower than that on circulating NK cells $(50.6 \pm 10.5 \% ; P=0.023$; Fig. 3$)$.

\section{Restoration of NKG2D expression on NK cells}

NKG2D expression on NK cells obtained from patients was restored after $48 \mathrm{~h}$ in culture with RPMI containing 10\% AB serum (Fig. 4). A similar phenomenon was observed in the clinic, in that NKG2D expression was restored on NK cells after surgery; values before and after surgery were $41.5 \pm 15.3 \%$ and $55.6 \pm 9.8 \%(n=10)$, respectively, and the difference was statistically significant $(P=0.036$; Fig. 5).
Fig. 1 a Representative result showing NKG2D expression on circulating natural killer (NK) cells in healthy controls and gastric cancer patients, determined by fluorescent activated cell sorting (FACS) ${ }^{\circledR}$. b NKG2D expression on circulating NK cells in gastric cancer patients was significantly lower than that in healthy controls $(P=0.019)$ (a) Healthy control

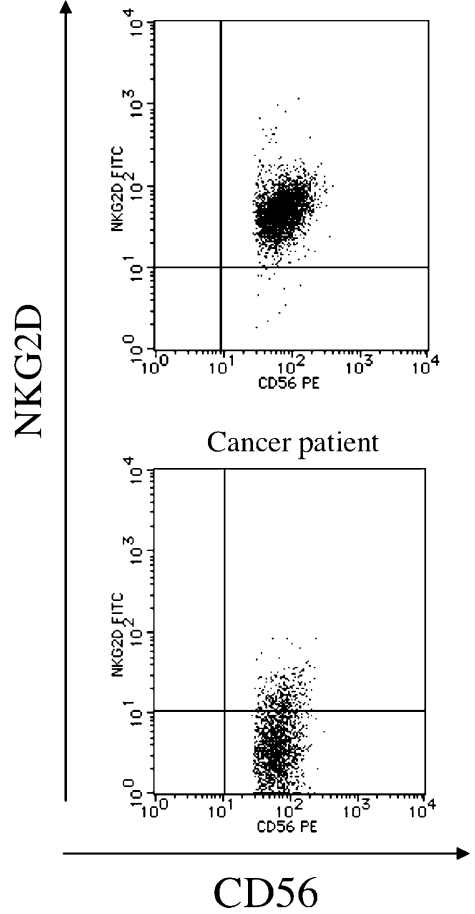

(b)

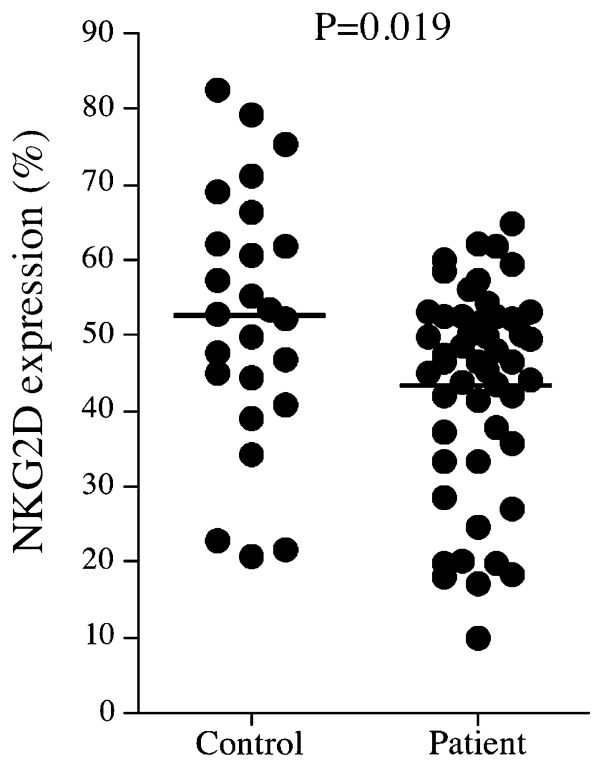


sMICA is associated with the downregulation of NKG2D expression on NK cells in cancer patients [28]. In the present study, the concentrations of sMICA in the sera

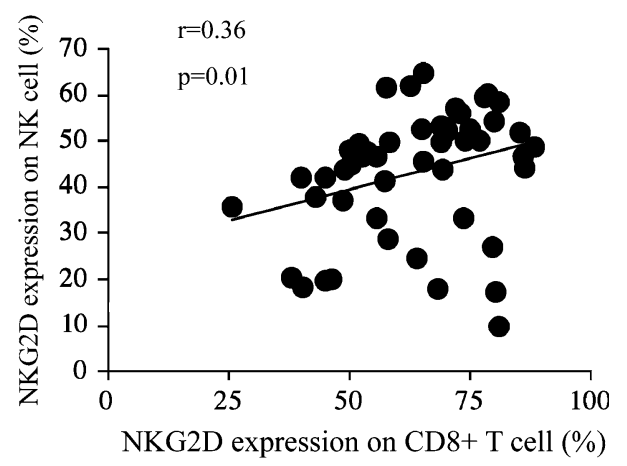

Fig. 2 There was a significant correlation between NKG2D expression on CD8 $+\mathrm{T}$ cells and NK cells $(r=0.36, P=0.01)$

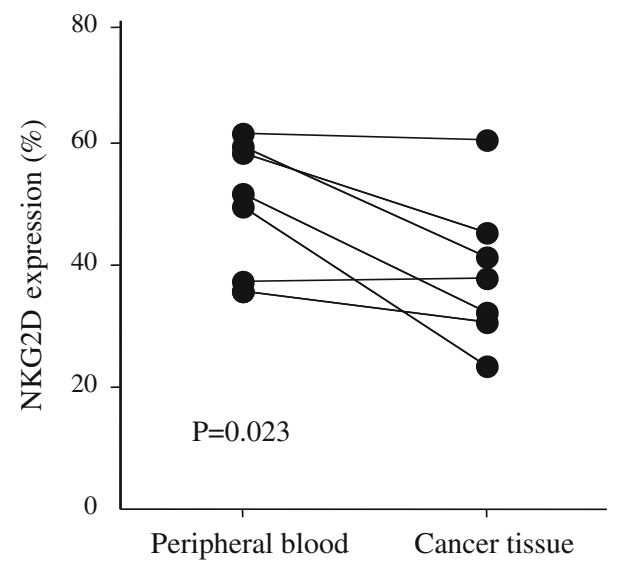

Fig. 3 NKG2D expression on NK cells in gastric cancer tissues $(38.8 \pm 12.1 \%)$ was significantly lower than that on circulating NK cells $(50.6 \pm 10.5 \% ; P=0.023)$ obtained from healthy controls and gastric cancer patients were $71.9 \pm 57.7$ and $86.0 \pm 121.6 \mathrm{pg} / \mathrm{ml}$, respectively. Furthermore, the concentration of sMICA in the supernatants obtained from six gastric cancer cell lines (TMK-1, MKN-1, MKN-28, MKN-45, MKN-74, and KATO-3) was $150.7 \pm 39.1 \mathrm{pg} / \mathrm{ml}$. There were no significant differences in the concentrations of sMICA among the healthy controls, cancer patients, and the supernatants from the gastric cancer cell lines.

\section{Discussion}

NK cells use inhibitory receptors to detect the absence of constitutively expressed "self" molecules on susceptible target cells. In particular, NK cells express MHC class I-specific receptors and 'lose' inhibitory signals when encountering MHC class I-deficient hematopoietic cells in several in vitro and in vivo models [29, 30]. Since most cancer cells downregulate their expression of MHC class I molecules, tumor cells are recognized as NK cell targets [1]. In fact, NK cells may participate in tumor immune surveillance, particularly in leukemia [31], neuroblastoma [32], and gastrointestinal stromal tumors [33]. However, NK cell function is suppressed in gastric cancer patients, even though the expression MHC class I molecules on gastric cancer cells is downregulated [34]. This indicates that some other mechanism is responsible for the defects in NK cell function observed in gastric cancer patients.

NKG2D-mediated immune activation is triggered by an interaction with ligands $[22,24]$ such as the MHC class I chain-related molecules (MICs) MICA and MICB [25], and the UL16-binding protein (ULBP) family [35-37]. In NK cells, the activation signal mediated by NKG2D dominates the inhibitory signals mediated by MHC class I
Fig. 4 NKG2D expression on NK cells obtained from gastric cancer patients was restored after $48 \mathrm{~h}$ in culture with RPMI containing $10 \% \mathrm{AB}$ serum
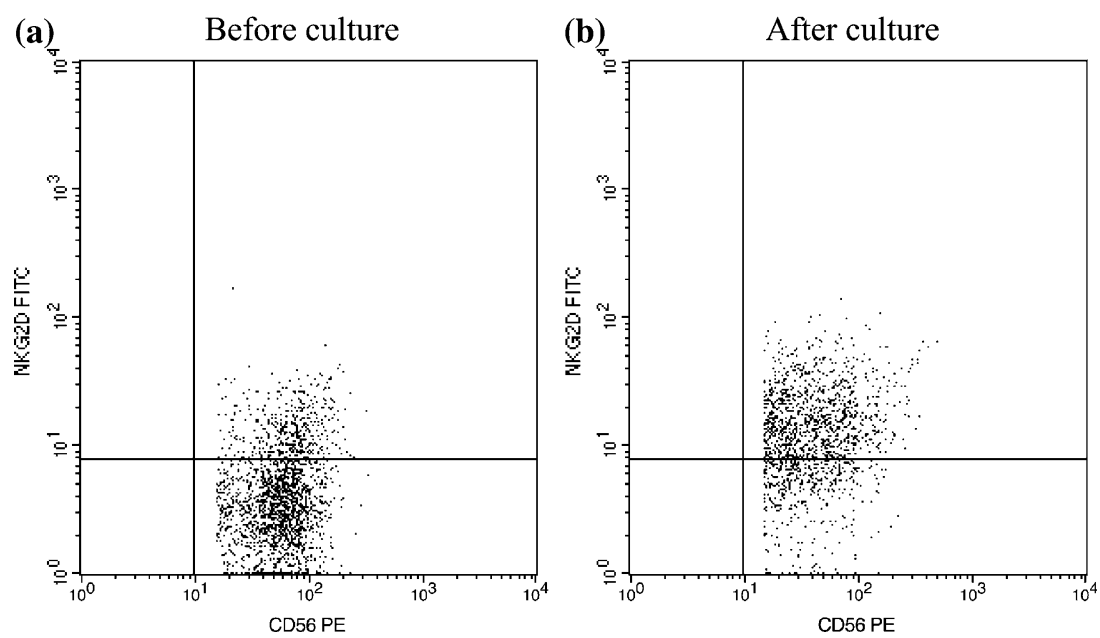


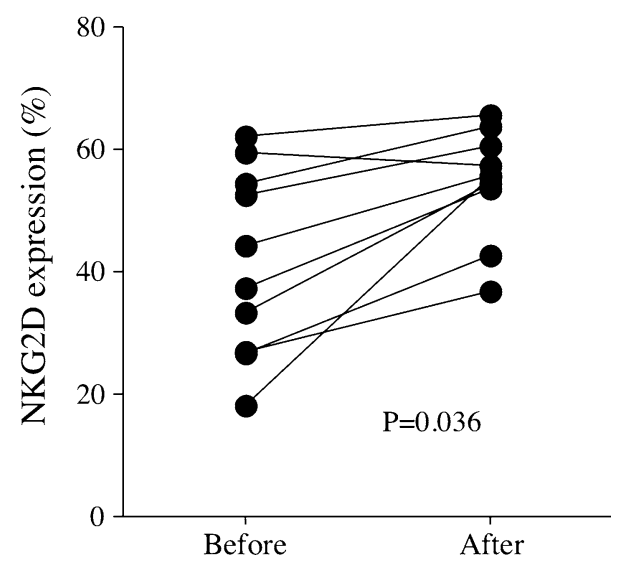

Fig. 5 NKG2D expression on NK cells before and after surgery was $41.5 \pm 15.3 \%$ and $55.6 \pm 9.8 \%(n=10)$, respectively, and the difference was statistically significant $(P=0.036)$

binding to killer inhibitory receptors, leading to the lysis of target cells that express NKG2D ligands [22-24]. This suggests that NKG2D engagement is extremely important for NK cell activation. In fact, it has been demonstrated that downregulation of NKG2D expression on NK cells is associated with impaired function of NK cells [28]. On the other hand, we have previously demonstrated that reduced NKG2D expression on CD8 $+\mathrm{T}$ cells is associated with reduced interferon (IFN)-gamma production by those cells, indicating that NK2GD engagement is also extremely important for the function of $\mathrm{CD} 8+\mathrm{T}$ cells [17]. In the present study, we showed that NKG2D expression by circulating NK cells in gastric cancer patients was significantly lower than that in healthy controls. Furthermore, reduced NKG2D expression on NK cells was closely correlated with disease severity. On the other hand, in the present study, there were no direct data indicating a correlation between decreased NKG2D expression on $\mathrm{NK}$ cells and tumor progression. In this regard, Guerra et al. [38] recently demonstrated that NKG2D-deficient mice were defective in tumor surveillance in models of spontaneous malignancy due to the defect in NK cell function, indicating that decreased NKG2D expression on NK cells is closely associated with tumor progression. Therefore, it is likely that most NK cells observed in the present study were not able to kill cancer cells due to reduced NKG2D expression, suggesting that the suppression of NKG2D expression might be closely related to the suppression of NK cell function and tumor progression in patients with gastric cancer. To the best of our knowledge, this is the first report demonstrating reduced $\mathrm{NKG} 2 \mathrm{D}$ expression on $\mathrm{NK}$ cells in gastric cancer patients.

The detailed mechanisms underlying the downregulation of NKG2D expression remain unclear and are under intense investigation. In the present study, decreased
NKG2D expression by NK cells from patients was restored after $48 \mathrm{~h}$ in culture with RPMI containing $10 \%$ AB serum. Furthermore, restoration of NKG2D expression by circulating NK cells was observed after complete surgical removal of the tumor. These results indicate that the decreased levels of NKG2D expression by NK cells observed in the present study were induced by factors that are exclusive to patients with gastric cancer. In fact, NKG2D expression by NK cells in cancer tissues was significantly lower than that in those isolated from peripheral blood. One factor related to reductions in NKG2D expression is MICA, which is the ligand for NKG2D. Groh et al. [26] recently demonstrated that tumorinfiltrating and systemic NK cells and CD8 $+\mathrm{T}$ cells often express low levels of NKG2D and are functionally compromised in cancer patients with $\mathrm{MICA}+$ or $\mathrm{MICB}+$ tumors. This has been attributed to the trans-acting effects of soluble MICA and MICB cleaved from solid tumors and leukemias by a tumor-associated metalloproteinase [26, 39, 40]. The engagement of sMICA promotes NKG2D internalization and degradation. Wu et al. [28] also demonstrated that sMICA downregulated NKG2D expression on NK cells in patients with prostate cancer. In the present study, however, we found no difference in the concentrations of sMICA between healthy controls and gastric cancer patients. This disparity could be caused by the presence of anti-MICA autoantibodies, which have been found in cancer patients with anti-CTLA-4 therapy-induced autoimmunity, and these autoantibodies may affect the ELISA detection of sMICA [41]. In addition, or alternatively to MICA, other NKG2D ligands, such as its close relative MICB, or ULBP2 and ULBP3, which are normally expressed on $\mathrm{B}$ cells, might be related to the downregulation of NKG2D on NK cells in gastric cancer patients.

It is possible that the downregulation of NKG2D on $\mathrm{NK}$ cells might be associated with the bad nutritional status of gastric cancer patients. In this regard, we examined the correlation between NKG2D expression on NK cells and the concentration of serum albumin, which is known to be associated with nutritional status, and found that there was no significant correlation between them (data not shown). Furthermore, the upregulation of NKG2D expression on NK cells 1 month after surgery clearly shows that poor nutritional status is not associated with reduced NKG2D expression on NK cells, since most patients are not able to eat enough due to the reduced volume of the stomach during this period. On the other hand, some cytokines, such as IFN-alpha, IFN-beta, and interleukin (IL)-12, which are known to activate NK cells, might be correlated with the reduced NKG2D expression on NK cells, since it has been demonstrated that the serum levels of these cytokines are low in gastric cancer patients, especially those with faradvanced disease [42]. In this regard, in the present study 
we demonstrated that NKG2D expression on NK cells obtained from gastric cancer patients was restored after $48 \mathrm{~h}$ in culture with RPMI containing $10 \%$ AB serum, which does not contain IFN-alpha, IFN-beta, or IL-12. Therefore, it is reasonable to think that other factors rather than IFN-alpha, IFN-beta, and IL-12 are associated with the reduced NKG2D expression on $\mathrm{NK}$ cells in gastric cancer patients.

In conclusion, our data strongly suggest that NKG2D expression on NK cells is decreased in gastric cancer patients. Moreover, this decreased NKG2D expression is correlated with disease severity. Considering the extremely important role played by NKG2D in the activation of NK cells, decreased NKG2D expression may be one of the key mechanisms responsible for NK cell dysfunction in gastric cancer patients. However, the detailed mechanisms responsible for this downregulation remain unknown. Further investigation into the mechanisms leading to decreased NKG2D expression on NK cells is urgently required.

Acknowledgments This work was supported by a grant from the Ministry of Education, Culture, Sports, Science, and Technology, Japan.

\section{References}

1. Trinchieri G. Biology of natural killer cells. Adv Immunol. 1989;47:187-376.

2. Tsutsui S, Morita M, Kuwano H, Matsuda H, Mori M, Okamura $\mathrm{S}$, et al. Influence of preoperative treatment and surgical operation on immune function of patients with esophageal carcinoma. J Surg Oncol. 1992;49:176-81.

3. Nunn ME, Herberman RB. Natural cytotoxicity of mouse, rat, and human lymphocytes against heterologous target cells. J Natl Cancer Inst. 1979;62:765-71.

4. Vose BM, Moore M. Natural cytotoxicity in humans: susceptibility of freshly isolated tumor cells to lysis. J Natl Cancer Inst. 1980;65:257-63.

5. Uchida A, Yanagawa E. Natural killer cell activity and autologous tumor killing activity in cancer patients: overlapping involvement of effector cells as determined in two-target conjugate cytotoxicity assay. J Natl Cancer Inst. 1984;73:1093-100.

6. Vujanovic NL, Herberman RB, Maghazachi AA, Hiserodt JC. Lymphokine-activated killer cells in rats, III. A simple method for the purification of large granular lymphocytes and their rapid expansion and conversion into lymphokine-activated killer cells. J Exp Med. 1988;167:15-29.

7. Kiessling R, Klein E, Wigzell H. "Natural" killer cells in the mouse, I. Cytotoxic cells with specificity for mouse Moloney leukemia cells. Specificity and distribution according to genotype. Eur J Immunol. 1975;5:112-7.

8. Herberman RB, Nunn ME, Lavrin DH. Natural cytotoxic reactivity of mouse lymphoid cells against syngeneic acid allogeneic tumors, I. Distribution of reactivity and specificity. Int J Cancer. 1975;16:216-29.

9. Ames IH, Garcia AM, John PA, Litty CA, Farrell MA, Tomar RH. Decreased natural cytotoxicity in mice with high incidence of mammary adenocarcinoma. Clin Immunol Immunopathol. 1986;38:265-73.

10. Cook JL, Lewis AM Jr. Immunological surveillance against DNA-virus-transformed cells: correlations between natural killer cell cytolytic competence and tumor susceptibility of athymic rodents. J Virol. 1987;61:2155-61.

11. Talmadge JE, Meyers KM, Prieur DJ, Starkey JR. Role of NK cells in tumour growth and metastasis in beige mice. Nature. 1980;284:622-4.

12. Hanna N, Burton RC. Definitive evidence that natural killer (NK) cells inhibit experimental tumor metastases in vivo. J Immunol. 1981;127:1754-8.

13. Barlozzari T, Leonhardt J, Wiltrout RH, Herberman RB, Reynolds CW. Direct evidence for the role of LGL in the inhibition of experimental tumor metastases. J Immunol. 1985;134:2783-9.

14. Imai K, Matsuyama S, Miyake S, Suga K, Nakachi K. Natural cytotoxic activity of peripheral-blood lymphocytes and cancer incidence: an 11-year follow-up study of a general population. Lancet. 2000;356:1795-9.

15. Parkin DM, Bray F, Ferlay J, Pisani P. Global cancer statistics, 2002. CA Cancer J Clin. 2005;55:74-108.

16. Takahashi A, Kono K, Amemiya H, Iizuka H, Fujii H, Matsumoto Y. Elevated caspase-3 activity in peripheral blood T cells coexists with increased degree of T-cell apoptosis and downregulation of TCR zeta molecules in patients with gastric cancer. Clin Cancer Res. 2001;7:74-80.

17. Osaki T, Saito H, Yoshikawa T, Matsumoto S, Tatebe S, Tsujitani $\mathrm{S}$, et al. Decreased NKG2D expression on CD8+ T cell is involved in immune evasion in patients with gastric cancer. Clin Cancer Res. 2007;13:382-7.

18. Sasada T, Kimura M, Yoshida Y, Kanai M, Takabayashi A. $\mathrm{CD} 4+\mathrm{CD} 25+$ regulatory $\mathrm{T}$ cells in patients with gastrointestinal malignancies: possible involvement of regulatory $\mathrm{T}$ cells in disease progression. Cancer. 2003;98:1089-99.

19. Oka M, Mitsunaga H, Hazama S, Yoshino S, Suzuki T. Natural killer activity and serum immunosuppressive acidic protein levels in esophageal and gastric cancers. Surg Today. 1993;23:669-74.

20. Takeuchi H, Maehara Y, Tokunaga E, Koga T, Kakeji Y, Sugimachi K. Prognostic significance of natural killer cell activity in patients with gastric carcinoma: a multivariate analysis. Am J Gastroenterol. 2001;96:574-8.

21. Glienke J, Sobanov Y, Brostjan C, Steffens C, Nguyen C, Lehrach $\mathrm{H}$, et al. The genomic organization of NKG2C, E, F, and D receptor genes in the human natural killer gene complex. Immunogenetics. 1998;48:163-73.

22. Raulet DH. Roles of the NKG2D immunoreceptor and its ligands. Nat Rev Immunol. 2003;3:781-90.

23. Watzl C. The NKG2D receptor and its ligands-recognition beyond the "missing self"? Microbes Infect. 2003;5:31-7.

24. Vivier E, Tomasello E, Paul P. Lymphocyte activation via NKG2D: towards a new paradigm in immune recognition? Curr Opin Immunol. 2002;14:306-11.

25. Bauer S, Groh V, Wu J, Steinle A, Phillips JH, Lanier LL, et al. Activation of NK cells and T cells by NKG2D, a receptor for stress-inducible MICA. Science. 1999;285:727-9.

26. Groh V, Wu J, Yee C, Spies T. Tumour-derived soluble MIC ligands impair expression of NKG2D and T-cell activation. Nature. 2002;419:734-8.

27. Japanese Gastric Cancer Association. Japanese classification of gastric carcinoma, 2nd English edition. Gastric Cancer. 1998;1:10-24.

28. Wu JD, Higgins LM, Steinle A, Cosman D, Haugk K, Plymate SR. Prevalent expression of the immunostimulatory MHC class I chain-related molecule is counteracted by shedding in prostate cancer. J Clin Invest. 2004;114:560-8. 
29. Yokoyama WM, Plougastel BF. Immune functions encoded by the natural killer gene complex. Nat Rev Immunol. 2003;3:304-16.

30. Parham P. MHC class I molecules and KIRs in human history, health and survival. Nat Rev Immunol. 2005;5:201-14.

31. Ruggeri L, Aversa F, Martelli MF, Velardi A. Allogeneic hematopoietic transplantation and natural killer cell recognition of missing self. Immunol Rev. 2006;214:202-18.

32. Castriconi R, Dondero A, Corrias MV, Lanino E, Pende D, Moretta L, et al. Natural killer cell-mediated killing of freshly isolated neuroblastoma cells: critical role of DNAX accessory molecule-1-poliovirus receptor interaction. Cancer Res. 2004;64:9180-4.

33. Borg C, Terme M, Taieb J, Menard C, Flament C, Robert C, et al. Novel mode of action of c-kit tyrosine kinase inhibitors leading to NK cell-dependent antitumor effects. J Clin Invest. 2004;114:379-88.

34. Shen YQ, Zhang JQ, Miao FQ, Zhang JM, Jiang Q, Chen H, et al. Relationship between the downregulation of HLA class I antigen and clinicopathological significance in gastric cancer. World $\mathbf{J}$ Gastroenterol. 2005;11:3628-31.

35. Cosman D, Mullberg J, Sutherland CL, Chin W, Armitage R, Fanslow W, et al. ULBPs, novel MHC class I-related molecules, bind to CMV glycoprotein UL16 and stimulate NK cytotoxicity through the NKG2D receptor. Immunity. 2001;14:123-33.
36. Chalupny NJ, Sutherland CL, Lawrence WA, Rein-Weston A, Cosman D. ULBP4 is a novel ligand for human NKG2D. Biochem Biophys Res Commun. 2003;305:129-35.

37. Conejo-Garcia JR, Benencia F, Courreges MC, Khang E, Zhang L, Mohamed-Hadley A, et al. Letal, A tumor-associated NKG2D immunoreceptor ligand, induces activation and expansion of effector immune cells. Cancer Biol Ther. 2003;2:446-51.

38. Guerra N, Tan YX, Joncker NT, Choy A, Gallardo F, Xiong N, et al. NKG2D-deficient mice are defective in tumor surveillance in models of spontaneous malignancy. Immunity. 2008;28:571-80.

39. Salih HR, Antropius H, Gieseke F, Lutz SZ, Kanz L, Rammensee $\mathrm{HG}$, et al. Functional expression and release of ligands for the activating immunoreceptor NKG2D in leukemia. Blood. 2003;102:1389-96.

40. Doubrovina ES, Doubrovin MM, Vider E, Sisson RB, O'Reilly RJ, Dupont B, et al. Evasion from NK cell immunity by MHC class I chain-related molecules expressing colon adenocarcinoma. J Immunol. 2003;171:6891-9.

41. Jinushi M, Hodi FS, Dranoff G. Therapy-induced antibodies to MHC class I chain-related protein A antagonize immune suppression and stimulate antitumor cytotoxicity. Proc Natl Acad Sci USA. 2006;103:9190-5.

42. Murakami S, Okubo K, Tsuji Y, Sakata H, Hamada S, Hirayama R. Serum interleukin-12 levels in patients with gastric cancer. Surg Today. 2004;34:1014-9. 